


\title{
Algoritmo para banho no leito no infarto agudo do miocárdio: estudo metodológico
}

\author{
Viviane de Moraes Sptiz ${ }^{1,2}$, Silvia Regina Martins dos Santos ${ }^{1,3,4}$, \\ Anna Beatriz Alves Pereira Lôbo ${ }^{1,5}$, Fernanda Faria Reis ${ }^{1,5}$, \\ Lucélia dos Santos Silva ${ }^{1,6}$, Dalmo Valério Machado de Lima ${ }^{1}$ \\ 'Universidade Federal Fluminense \\ ${ }^{2}$ Secretaria de Saúde do Estado do Rio de Janeiro \\ ${ }^{3}$ Universidade do Estado do Rio de Janeiro \\ ${ }^{4}$ Universidade Veiga de Almeida \\ ${ }^{5}$ Hospital Estadual Alberto Torres \\ ${ }^{6}$ Secretaria Municipal de Saúde do Rio de Janeiro
}

\section{RESUMO}

Objetivo: pretende-se construir um algoritmo para indicação do banho no leito no paciente adulto internado com infarto agudo do miocárdio. Método: Estudo metodológico realizado entre março de 2015 e janeiro de 2016, com base na revisão da literatura e nas bases de dados PUBMED, LILACS, WEB OF SCIENCE, CINAHL e COCHRANE, com recorte temporal de 2010 a 2015 e dados de um ensaio clínico realizado de junho a novembro de 2015, em um hospital privado de Niterói, com 19 pacientes adultos. A análise estatística será feita por meio de correlação e regressão logística das variáveis: tempo e horário do banho, temperatura da água, tipo de infarto, posicionamento, frequência cardíaca, pressão arterial, saturação periférica de oxigênio, consumo de oxigênio pelo miocárdico e volume sistólico. Para construção do algoritmo, se utilizará o freeware Bizagi BPMN Modeler versão 3.0. A validação do conteúdo será realizada mediante reunião com especialistas, recrutados através da Plataforma Lattes.

Descritores: Banhos; Infarto do Miocárdio; Algoritmos; Hemodinâmica; Oximetria. 


\section{INTRODUÇÃO}

O banho no leito é uma atividade exclusiva da enfermagem e faz parte dos cuidados cotidianos prestados aos pacientes internados na Unidades de Terapia Intensiva. Em uma pesquisa em que se avaliou o impacto do banho no leito sobre o tempo de desmame de pacientes em ventilação mecânica, foi evidenciado que o banho não aumentou o tempo de desmame, contradizendo a expectativa da prática clínica. Atribuíram-se, como fator positivo, a presença da enfermeira, sugerindo que, durante o banho, o paciente recebe maior atenção e conforto, o que promove bem-estar e influencia positivamente na recuperação do doente ${ }^{(1)}$. Porém, o banho no leito pode desencadear algumas alterações fisiológicas. Um estudo que avaliou as repercussões oxi-hemodinâmicas nos pacientes em pós-operatório de cirurgia cardíaca evidenciou alterações estatisticamente significativas (2) na frequência cardíaca e na pressão arterial sistólica.

Nas últimas décadas, surgiu uma grande preocupação com a segurança do paciente. Ao realizar uma busca nas principais bases de dados, nos últimos cinco anos, constataram-se diminuto material científico e ausência de uma ferramenta de tomada de decisão para a prática de banho no leito no paciente infartado, justificando a presente proposta. O algoritmo de banho subsidiará uma prática assistencial com qualidade a fim de promover a segurança do paciente e de minimizar fatores agravantes.

\section{HIPÓTESE}

Um algoritmo de avaliação pré-banho, aplicado pelo enfermeiro, auxiliará a tomada de decisão de forma a garantir que esse banho seja dado de forma segura nos pacientes infartados.

\section{OBJETIVO}

Construir algoritmo para indicação do banho no leito no paciente adulto internado com infarto agudo do miocárdio.

\section{MÉTODO}

Este estudo faz parte das atividades do Mestrado Profissional em Enfermagem Assistencial da Universidade Federal Fluminense. Trata-se de um estudo metodológico desenvolvido mediante dados coletados de um ensaio clínico randomizado não controlado, desenvolvido na Unidade Coronariana de um hospital privado de Niterói, no período de junho a novembro de 2015, e mediante a revisão de literatura nas bases de dados PUBMED, LILACS, WEB OF SCIENCE, CINAHL e COCHRANE com recorte temporal de 2010 a 2015, utilizando os descritores Banhos, Infarto do miocárdio, Algoritmos, Hemodinâmica e Oximetria no período de março de 2015 a janeiro 2016. Foram selecionados dezoito artigos sobre banho no leito na terapia intensiva para o embasamento teórico da pesquisa.

O ensaio clínico constituiu uma amostra de conveniência de 19 pacientes adultos infartados, submetidos a dois banhos no leito em dias consecutivos, um com controle de temperatura da água constante a $40^{\circ}$ e um sem constância na temperatura da água. Ambos foram monitorizados pelo ICG CardioScreem 2000 MEDIS. O critério de inclusão nesse ensaio clínico foi: pacientes adultos, com infarto do miocárdio classificação Killip-Kimball I e 
II em até 48 horas do início do evento, que tivessem completado os dois banhos. Os critérios de exclusão foram: pacientes em pós-operatório de cirurgia cardíaca, com insuficiência cardíaca crônica, com tempo de banho superior a $20 \mathrm{~min}$ e com patologias que pudessem comprometer a acurácia da ICG como choque séptico, problemas na válvula aórtica ou presença de prótese aórtica, hipertensão grave, taquicardia (acima de 200bpm), altura inferior a $120 \mathrm{~cm}$ ou superior a $230 \mathrm{~cm}$, peso inferior a $30 \mathrm{Kg}$ ou superior a $155 \mathrm{Kg}$, presença de balão intra-aórtico.

Subsidiarão a construção do algoritmo dados da literatura e procedentes do ensaio clínico, e será utilizado o freeware Bizagi BPMN Modeler versão 3.0.

Serão utilizados dados sociodemográficos da saúde atual e pregressa para a análise do perfil da amostra. As variáveis independentes serão tempo de banho, temperatura da água, horário de banho, tipo de infarto e posicionamento, e as variáveis dependentes, frequência cardíaca, pressão arterial e média, saturação periférica de oxigênio, consumo de oxigênio pelo miocárdico e volume sistólico. Para a análise estatística, serão efetuados os testes de correlação e regressão logística multivariada.

Para validação do conteúdo do algoritmo, será formado um comitê de especialistas a partir de uma busca curricular na Plataforma Lattes do CNPq. Os critérios de inclusão serão: doutores com nacionalidade Brasileira que publicaram trabalhos ou pesquisaram sobre banho no leito, cardiointensivismo, infarto agudo do miocárdio e estudos de alterações hemodinâmicas durante o banho, sistematização da assistência de enfermagem, segurança do paciente e profissionais não doutores que tiverem experiência em clínica assistencial. Tomando como base o artigo de Alexandre e Coluci $^{(3)}$, serão recrutados cinco especialistas para compor o comitê.

Será enviado aos especialistas um questionário por meio eletrônico, utilizando a Técnica Delphi, com afirmações sobre o conteúdo que deverá compor o algoritmo. Após devolução das respostas, será efetuada a avaliação de concordância entre elas por meio do Índice de Concordância Kappa.

\section{REFERÊNCIAS}

1. Sereika SM, Tate JA, DiVirgilio-Thomas D, et al. The association between bathing and weaning trial duration. Heart Lung. [Internet]. 2011 Jan-Feb [cited 2016 Oct. 20];40(1):41-8. Available from: doi: https://www.ncbi.nlm.nih. gov/pmc/articles/PMC2997168/_doi: 10.1016 / j.hrtlng.2010.03.005.

2. Madrid SQ, López CC, Otálvaro AFT, Padilla LMR. Alteraciones hemodinámicas del paciente crítico cardiovascular durante la realización del baño diario. Medicina U.P.B. .[Internet]. 2012 Ene-Jun [cited 2016 October 20] 31(1):1926. Available from: http://www.redalyc. org:9081/articulo.oa?id=159024332004

3. Alexandre, NMC, Coluci MZO. Validade de conteúdo nos processos de construção e adaptação de instrumentos de medidas. Ciênc. saúde coletiva. [Internet]. 2011 July [cited 2016 Jan 27] 16(7):3061-68. Available from: http://www. scielo.br/scielo.php?script=sci_arttext\&pid $=$ S1413-81232011000800006 http://dx.doi. org/10.1590/S1413-81232011000800006. 
Todos os autores participaram das fases dessa publicação em uma ou mais etapas a seguir, de acordo com as recomendações do International Committe of Medical Journal Editors (ICMJE, 2013): (a) participação substancial na concepção ou confecção do manuscrito ou da coleta, análise ou interpretação dos dados; (b) elaboração do trabalho ou realização de revisão crítica do conteúdo intelectual; (c) aprovação da versão submetida. Todos os autores declaram para os devidos fins que são de suas responsabilidades o conteúdo relacionado a todos os aspectos do manuscrito submetido ao OBJN. Garantem que as questões relacionadas com a exatidão ou integridade de qualquer parte do artigo foram devidamente investigadas e resolvidas. Eximindo, portanto o OBJN de qualquer participação solidária em eventuais imbróglios sobre a materia em apreço. Todos os autores declaram que não possuem conflito de interesses, seja de ordem financeira ou de relacionamento, que influencie a redação e/ ou interpretação dos achados. Essa declaração foi assinada digitalmente por todos os autores conforme recomendação do ICMJE, cujo modelo está disponível em http://www.objnursing.uff.br/normas/DUDE_ final_13-06-2013.pdf
Recebido: 08/09/2016

Revisado: 31/10/2016

Aprovado:31/10/2016 